NCKU-HEP-99-01

IRB-TH-1/99

hep-ph/9902205

\title{
Determination of heavy-meson wave functions from $B$ decays
}

\author{
Hsiang-nan $\operatorname{Li}^{a}$ and Blaženka Melić ${ }^{b}$ \\ ${ }^{a}$ Department of Physics, National Cheng-Kung University, \\ Tainan, Taiwan 701, Republic of China \\ ${ }^{b}$ Theoretical Physics Division, Rudjer Bošković Institute \\ P.O. Box 1016, HR-10001 Zagreb, Croatia
}

\begin{abstract}
We extract the $B, D$, and $D^{*}$ meson wave functions from the CLEO data of the decays $B \rightarrow K^{*} \gamma$ and $B \rightarrow D^{(*)} \pi$ in the perturbative QCD framework. In this formalism, various logarithmic corrections are organized to give the Wilson evolution from the $W$ boson mass down to the characteristic scale of a decay process, which is of order of the $b$ quark mass, and the Sudakov evolution from the characteristic scale to a lower factorization scale of order $\Lambda_{\mathrm{QCD}}$. With large logarithms organized, the $b$ quark decay amplitudes are evaluated reliably in perturbation theory. Below the factorization scale, QCD dynamics is regarded as being nonperturbative, and absorbed into meson wave functions. Because of their universality, the heavy-meson wave functions determined in this work, can be employed to make predictions of other
\end{abstract}


decay modes. We also observe that the dependence of heavy meson wave functions on intrinsic parton transverse momenta plays an important role in the explanation of data. 


\section{INTRODUCTION}

Recently, a PQCD factorization theorem for exclusive heavy-meson decays has been developed by one of us [四, and applied to the nonleptonic $B \rightarrow D^{(*)} \pi(\rho)$ decays [2] and the penguin-induced radiative $B \rightarrow K^{*} \gamma$ decays [3]. These decays involve three scales: the $W$ boson mass $M_{W}$, at which the matching conditions of the effective weak Hamiltonian to the full Hamiltonian are defined, the typical scale $t$ of a hard amplitude, which reflects the specific dynamics of a heavy-meson decay, and the factorization scale $1 / b$, with $b$ being the conjugate variable of parton transverse momenta. The dynamics below $1 / b$ is regarded as being completely nonperturbative, and parametrized into meson wave functions $\phi(x, b), x$ being the momentum fraction. Above the factorization scale, PQCD is reliable and radiative corrections produce two types of large logarithms: $\ln \left(M_{W} / t\right)$ and $\ln (t b)$. The former are summed by renormalization-group ( $\mathrm{RG}$ ) equations to give the evolution from $M_{W}$ down to $t$ described by the Wilson coefficients $c(t)$, while the latter are summed to give the evolution from $t$ to $1 / b$.

There also exist double logarithms $\ln ^{2}(P b)$ from the overlap of collinear and soft divergences in radiative corrections to meson wave functions, $P$ being the dominant light-cone component of a meson momentum. The resummation of these double logarithms leads to a Sudakov form factor $\exp [-s(P, b)]$, which suppresses the long-distance contributions in the large $b$ region, and vanishes as $b=1 / \Lambda, \Lambda \equiv \Lambda_{\mathrm{QCD}}$ being the QCD scale. This factor guarantees the applicability of PQCD around the energy scale of the $b$ quark mass, which is our motivation to develop a PQCD formalism for heavy-meson decays. For a detailed derivation of the relevant Sudakov form factors, we refer the readers to [4,5]. With all the large logarithms organized, the remaining finite contributions are absorbed into the hard $b$ quark decay amplitude $H(t)$.

We do not claim that contributions to $B$ meson decays are purely perturbative, but that perturbative contributions are important, and it makes sense to compare PQCD predictions with experimental data. It has been shown that about $70 \%$ of the full contribution to the 
decay $B \rightarrow D \pi$ arises from the region with $\alpha_{s}<1\left(\alpha_{s} / \pi<0.3\right)$ in the above PQCD formalism [5]. Since energy releases involved in $B$ meson decays are located in the transition region from nonperturbative to perturbative QCD according to the analysis in [6], nonperturbative approaches, such as QCD sum rules [7], also give sizeable contributions. In fact, $B$ meson decays are a subject on which perturbative and nonperturbative approaches complement each other.

A three-scale factorization formula for exclusive $B$ meson decays possesses the typical expression,

$$
c(t) \otimes H(t) \otimes \phi(x, b) \otimes \exp \left[-s(P, b)-2 \int_{1 / b}^{t} \frac{d \bar{\mu}}{\bar{\mu}} \gamma\left(\alpha_{s}(\bar{\mu})\right)\right],
$$

where the exponential involving the quark anomalous dimension $\gamma=-\alpha_{s} / \pi$ describes the evolution from $t$ to $1 / b$ mentioned above. All the convolution factors in the above formula, except for the wave functions $\phi(x, b)$, are calculable in perturbation theory. The wave functions, though not calculable, are universal, since they absorb the long-distance dynamics, which is insensitive to the specific decay of the $b$ quark into light quarks with large energy release. The universality of nonperturbative wave functions is the fundamental concept of PQCD factorization theorems. It can be proved rigorously, but the proof is not the point of this work, and will not be supplied here.

Because of the universality, the strategy of PQCD factorization theorems is as follows: evaluate all perturbative factors for some decay modes, and adjust the wave functions such that predictions from the corresponding factorization formulas match experimental data. At this stage, the nonperturbative wave functions are determined up to the twists and orders of the coupling constant, at which the factorization formulas are constructed. Then evaluate all the perturbative factors for another decay mode. Input the extracted wave functions into the factorization formulas of the same twist and orders, and make predictions. With this strategy, PQCD factorization theorems are model independent and possess a predictive power.

For light-meson wave functions, such as those of $\pi, \rho$, and $K^{(*)}$ mesons, various ex- 
perimental data and QCD sum rules 8 10 have provided reliable information. For heavy mesons, such as $B$ and $D^{(*)}$, the knowledge is still poor. In this paper we shall determine the heavy meson wave functions from the available experimental data. Since two-body nonleptonic decays involve the maximal energy release that guarantees the applicability of PQCD, and their data are abundant, the decays $B \rightarrow D^{(*)} \pi$ are ideal processes for extracting the $B$ and $D^{(*)}$ meson wave functions. However, both of the $B$ and $D^{(*)}$ wave functions are unknown, and thus we need the additional data of the $B \rightarrow K^{*} \gamma$ decay 11] in order to determine the $B$ meson wave function first. After determining the $B$ meson wave function, we apply it to the analysis of the $B \rightarrow D^{(*)} \pi$ decays and obtain the $D$ and $D^{*}$ meson wave functions.

The factorization formulas for the $B \rightarrow K^{*} \gamma$ and $B \rightarrow D^{(*)} \pi$ decays are presented in Sec. II and Sec. III, respectively. The numerical analysis is performed in Sec. IV. Section V is the conclusion.

\section{THE $B \rightarrow K^{*} \gamma$ DECAY}

The effective Hamiltonian for the flavor-changing $b \rightarrow s$ transition is given by [12]

$$
H_{\mathrm{eff}}=-\frac{G_{F}}{\sqrt{2}} V_{t s}^{*} V_{t b} \sum_{i=1}^{8} C_{i}(\mu) O_{i}(\mu)
$$

with

$$
\begin{aligned}
O_{1} & =\left(\bar{s}_{i} c_{j}\right)_{V-A}\left(\bar{c}_{j} b_{i}\right)_{V-A}, \quad O_{2}=\left(\bar{s}_{i} c_{i}\right)_{V-A}\left(\bar{c}_{j} b_{j}\right)_{V-A}, \\
O_{3} & =\left(\bar{s}_{i} b_{i}\right)_{V-A} \sum_{q}\left(\bar{q}_{j} q_{j}\right)_{V-A}, \quad O_{4}=\left(\bar{s}_{i} b_{j}\right)_{V-A} \sum_{q}\left(\bar{q}_{j} q_{i}\right)_{V-A}, \\
O_{5} & =\left(\bar{s}_{i} b_{i}\right)_{V-A} \sum_{q}\left(\bar{q}_{j} q_{j}\right)_{V+A}, \quad O_{6}=\left(\bar{s}_{i} b_{j}\right)_{V-A} \sum_{q}\left(\bar{q}_{j} q_{i}\right)_{V+A}, \\
O_{7} & =\frac{e}{4 \pi^{2}} \bar{s}_{i} \sigma^{\mu \nu}\left(m_{s} P_{L}+m_{b} P_{R}\right) b_{i} F_{\mu \nu}, \\
O_{8} & =-\frac{g}{4 \pi^{2}} \bar{s}_{i} \sigma^{\mu \nu}\left(m_{s} P_{L}+m_{b} P_{R}\right) T_{i j}^{a} b_{j} G_{\mu \nu}^{a},
\end{aligned}
$$

$i, j$ being the color indices. It has been observed that the contributions to the $B \rightarrow K^{*} \gamma$ decay rate come mainly from the operators $\mathrm{O}_{2}, \mathrm{O}_{7}$ and $\mathrm{O}_{8}$ [3]. $\mathrm{O}_{2}$ contributes via a charm- 
quark loop, from which the photon and an off-shell gluon are radiated. This off-shell gluon is then reabsorbed by the spectator quark in the $B$ or $K^{*}$ meson. This $b \rightarrow s g^{*} \gamma$ vertex has been computed in [3, [13].

The momenta of the $B$ and $K^{*}$ mesons in light-cone coordinates are written as $P_{1}=$ $\left(M_{B} / \sqrt{2}\right)\left(1,1, \mathbf{0}_{T}\right)$ and $P_{2}=\left(M_{B} / \sqrt{2}\right)\left(1, r^{2}, \mathbf{0}_{T}\right)$, respectively, with $r=M_{K^{*}} / M_{B}$. The $B$ meson is at rest with the above parametrization of momenta. We define the momenta of light valence quarks in the $B$ and $K^{*}$ mesons as $k_{1}$ and $k_{2}$, respectively. $k_{1}$ has a minus component $k_{1}^{-}$, giving the momentum fraction $x_{1}=k_{1}^{-} / P_{1}^{-}$, and small transverse components $\mathbf{k}_{1 T} \cdot k_{2}$ has a large plus component $k_{2}^{+}$, giving $x_{2}=k_{2}^{+} / P_{2}^{+}$, and small $\mathbf{k}_{2 T}$. The photon momentum is then $P_{3}=P_{1}-P_{2}$, whose nonvanishing component is only $P_{3}^{-}$.

The $B \rightarrow K^{*} \gamma$ decay amplitude can be decomposed as

$$
M=\epsilon_{\gamma}^{*} \cdot \epsilon_{K^{*}}^{*} M^{S}+i \epsilon_{\mu \rho+-} \epsilon_{\gamma}^{* \mu} \epsilon_{K^{*}}^{* \rho} M^{P}
$$

with $\epsilon_{\gamma}$ and $\epsilon_{K^{*}}$ the polarization vectors of the photon and of the $K^{*}$ meson, respectively. The total rate of the $B \rightarrow K^{*} \gamma$ decay is given by

$$
\Gamma=\frac{1-r^{2}}{8 \pi M_{B}}\left(\left|M^{S}\right|^{2}+\left|M^{P}\right|^{2}\right)
$$

We further decompose $M^{S}$ and $M^{P}$ as

$$
M^{i}=M_{2}^{i}+M_{7}^{i}+M_{8}^{i}
$$

where $i=S$ or $P$, and the terms on the right-hand side represent the contributions from the operators $\mathrm{O}_{2}, \mathrm{O}_{7}$, and $\mathrm{O}_{8}$, respectively.

The Sudakov resummation of the large logarithmic corrections to the $B$ meson wave function $\phi_{B}$ and to the $K^{*}$ meson wave function $\phi_{K^{*}}$ leads to the exponentials $\exp \left(-S_{B}\right)$ and $\exp \left(-S_{K^{*}}\right)$, respectively, with the exponents

$$
\begin{aligned}
S_{B}(t) & =s\left(x_{1} P_{1}^{-}, b_{1}\right)+2 \int_{1 / b_{1}}^{t} \frac{d \bar{\mu}}{\bar{\mu}} \gamma\left(\alpha_{s}(\bar{\mu})\right), \\
S_{K^{*}}(t) & =s\left(x_{2} P_{2}^{+}, b_{2}\right)+s\left(\left(1-x_{2}\right) P_{2}^{+}, b_{2}\right)+2 \int_{1 / b_{2}}^{t} \frac{d \bar{\mu}}{\bar{\mu}} \gamma\left(\alpha_{s}(\bar{\mu})\right) .
\end{aligned}
$$


The variable $b_{1}\left(b_{2}\right)$, conjugate to the parton transverse momentum $k_{1 T}\left(k_{2 T}\right)$, represents the transverse extent of the $B\left(K^{*}\right)$ meson. The exponent $s$ is [14, 15.

$$
s(Q, b)=\int_{1 / b}^{Q} \frac{d \mu}{\mu}\left[\ln \left(\frac{Q}{\mu}\right) A\left(\alpha_{s}(\mu)\right)+B\left(\alpha_{s}(\mu)\right)\right] \text {, }
$$

where the anomalous dimensions $A$ to two loops and $B$ to one loop are

$$
\begin{aligned}
& A=C_{F} \frac{\alpha_{s}}{\pi}+\left[\frac{67}{9}-\frac{\pi^{2}}{3}-\frac{10}{27} n_{f}+\frac{2}{3} \beta_{0} \ln \left(\frac{e^{\gamma_{E}}}{2}\right)\right]\left(\frac{\alpha_{s}}{\pi}\right)^{2}, \\
& B=\frac{2}{3} \frac{\alpha_{s}}{\pi} \ln \left(\frac{e^{2 \gamma_{E}-1}}{2}\right),
\end{aligned}
$$

with $C_{F}=4 / 3$ a color factor and $\gamma_{E}$ the Euler constant. The one-loop expression of the running coupling constant,

$$
\alpha_{s}(\mu)=\frac{4 \pi}{\beta_{0} \ln \left(\mu^{2} / \Lambda^{2}\right)}
$$

is substituted into Eq. (2.7) with the coefficient $\beta_{0}=\left(33-2 n_{f}\right) / 3$. The Sudakov factor in Eq. (2.6) suppresses long-distance contributions from the large $b$ region, and improves the applicability of PQCD to $B$ meson decays.

We quote the factorization formulas for $M_{l}^{i}$ [3] in terms of the overall factor

$$
\Gamma^{(0)}=\frac{G_{F}}{\sqrt{2}} \frac{e}{\pi} V_{t s}^{*} V_{t b} C_{F} M_{B}^{5}
$$

The amplitudes contributed by $\mathrm{O}_{2}$ are written as

$$
\begin{aligned}
M_{2}^{S}= & \Gamma^{(0)} \frac{4}{3} \int_{0}^{1} d x \int_{0}^{1-x} d y \int_{0}^{1} d x_{1} d x_{2} \int_{0}^{1 / \Lambda} b d b \phi_{B}\left(x_{1}, b\right) \phi_{K^{*}}\left(x_{2}\right) \\
& \times \alpha_{s}\left(t_{2}\right) c_{2}\left(t_{2}\right) \exp \left[-S_{B}\left(t_{2}\right)-S_{K^{*}}\left(t_{2}\right)\right]_{b_{1}=b_{2}=b} \\
& \times\left[\left(1-r^{2}+2 r x_{2}+2 x_{1}\right) y-\left(r x_{2}+3 x_{1}\right)(1-x)\right] \\
& \times \frac{(1-r)\left(1-r^{2}\right) x_{2} x}{x y\left(1-r^{2}\right) x_{2} M_{B}^{2}-M_{c}^{2}} H_{2}\left(A b, \sqrt{\left|B_{2}^{2}\right|} b\right), \\
M_{2}^{P}= & \Gamma^{(0)} \frac{4}{3} \int_{0}^{1} d x \int_{0}^{1-x} d y \int_{0}^{1} d x_{1} d x_{2} \int_{0}^{1 / \Lambda} b d b \phi_{B}\left(x_{1}, b\right) \phi_{K^{*}}\left(x_{2}\right) \\
& \times \alpha_{s}\left(t_{2}\right) c_{2}\left(t_{2}\right) \exp \left[-S_{B}\left(t_{2}\right)-S_{K^{*}}\left(t_{2}\right)\right]_{b_{1}=b_{2}=b}
\end{aligned}
$$




$$
\begin{aligned}
& \times\left[\left((1-r)\left(1-r^{2}\right)+2 r^{2} x_{2}+2 x_{1}\right) y\right. \\
& \left.-\left(r(1+r) x_{2}+(3-r) x_{1}\right)(1-x)\right] \\
& \times \frac{\left(1-r^{2}\right) x_{2} x}{x y\left(1-r^{2}\right) x_{2} M_{B}^{2}-M_{c}^{2}} H_{2}\left(A b, \sqrt{\left|B_{2}^{2}\right|} b\right),
\end{aligned}
$$

with

$$
\begin{aligned}
& A^{2}=x_{1} x_{2} M_{B}^{2} \\
& B_{2}^{2}=x_{1} x_{2} M_{B}^{2}-\frac{y}{1-x}\left(1-r^{2}\right) x_{2} M_{B}^{2}+\frac{M_{c}^{2}}{x(1-x)} \\
& t_{2}=\max \left(A, \sqrt{\left|B_{2}^{2}\right|}, 1 / b\right)
\end{aligned}
$$

and $M_{c}$ being the charm quark mass. $t_{2}$ is the characteristic scale of the hard amplitude

$$
\begin{aligned}
H_{2}\left(A b, \sqrt{\left|B_{2}^{2}\right|} b\right) & =K_{0}(A b)-K_{0}\left(\sqrt{\left|B_{2}^{2}\right|} b\right), \quad B_{2}^{2}>0 \\
& =K_{0}(A b)-i \frac{\pi}{2} H_{0}^{(1)}\left(\sqrt{\left|B_{2}^{2}\right|} b\right), \quad B_{2}^{2}<0 .
\end{aligned}
$$

The amplitudes from $O_{7}$ with a hard gluon attaching the quarks in the $B$ meson or in the $K^{*}$ meson are

$$
\begin{aligned}
M_{7}^{S}=-M_{7}^{P}= & \Gamma^{(0)} 2 \int_{0}^{1} d x_{1} d x_{2} \int_{0}^{1 / \Lambda} b_{1} d b_{1} b_{2} d b_{2} \phi_{B}\left(x_{1}, b_{1}\right) \phi_{K^{*}}\left(x_{2}\right) \\
& \times \alpha_{s}\left(t_{7}\right) C_{7}\left(t_{7}\right) \exp \left[-S_{B}\left(t_{7}\right)-S_{K^{*}}\left(t_{7}\right)\right] \\
& \times\left(1-r^{2}\right)\left\{r H_{7}^{(a)}\left(A b_{2}, \sqrt{\left|B_{7}^{2}\right|} b_{1}, \sqrt{\left|B_{7}^{2}\right|} b_{2}\right)\right. \\
& \left.+\left[1+r+(1-2 r) x_{2}\right] H_{7}^{(b)}\left(A b_{1}, C_{7} b_{1}, C_{7} b_{2}\right)\right\}
\end{aligned}
$$

with

$$
\begin{aligned}
& B_{7}^{2}=\left(x_{1}-r^{2}\right) m_{B}^{2}, \quad C_{7}^{2}=x_{2} m_{B}^{2} \\
& t_{7}=\max \left(A, \sqrt{\left|B_{7}^{2}\right|}, C_{7}, 1 / b_{1}, 1 / b_{2}\right)
\end{aligned}
$$

The hard functions are given by

$$
\begin{aligned}
H_{7}^{(a)}\left(A b_{2}, \sqrt{\left|B_{7}^{2}\right|} b_{1}, \sqrt{\left|B_{7}^{2}\right|} b_{2}\right)= \\
K_{0}\left(A b_{2}\right) h\left(\sqrt{\left|B_{7}^{2}\right|} b_{1}, \sqrt{\left|B_{7}^{2}\right|} b_{2}\right), \quad B_{7}^{2}>0, \\
K_{0}\left(A b_{2}\right) h^{\prime}\left(\sqrt{\left|B_{7}^{2}\right|} b_{1}, \sqrt{\left|B_{7}^{2}\right|} b_{2}\right), \quad B_{7}^{2}<0,
\end{aligned}
$$


with

$$
\begin{aligned}
h & =\theta\left(b_{1}-b_{2}\right) K_{0}\left(\sqrt{\left|B_{7}^{2}\right|} b_{1}\right) I_{0}\left(\sqrt{\left|B_{7}^{2}\right|} b_{2}\right)+\left(b_{1} \leftrightarrow b_{2}\right), \\
h^{\prime} & =i \frac{\pi}{2}\left[\theta\left(b_{1}-b_{2}\right) H_{0}^{(1)}\left(\sqrt{\left|B_{7}^{2}\right|} b_{1}\right) J_{0}\left(\sqrt{\left|B_{7}^{2}\right|} b_{2}\right)+\left(b_{1} \leftrightarrow b_{2}\right)\right],
\end{aligned}
$$

and

$$
H_{7}^{(b)}\left(A b_{1}, C_{7} b_{1}, C_{7} b_{2}\right)=K_{0}\left(A b_{1}\right) h\left(C_{7} b_{1}, C_{7} b_{2}\right)
$$

The relation $M_{7}^{S}=-M_{7}^{P}$ reflects the equality of the parity-conserving and parity-violating contributions induced by $O_{7}$.

The amplitudes from $O_{8}$, where the photon is radiated by each quark in the $B$ and $K^{*}$ mesons, are

$$
\begin{aligned}
& M_{8}^{S}=-\Gamma^{(0)} \frac{1}{3} \int_{0}^{1} d x_{1} d x_{2} \int_{0}^{1 / \Lambda} b_{1} d b_{1} b_{2} d b_{2} \phi_{B}\left(x_{1}, b_{1}\right) \phi_{K^{*}}\left(x_{2}\right) \\
& \times \alpha_{s}\left(t_{8}\right) C_{8}\left(t_{8}\right) \exp \left[-S_{B}\left(t_{8}\right)-S_{K^{*}}\left(t_{8}\right)\right] \\
& \times\left\{\left(1-r^{2}+x_{1}\right)\left(r x_{2}+x_{1}\right) H_{8}^{(a)}\left(A b_{2}, B_{8} b_{1}, B_{8} b_{2}\right)\right. \\
& +\left[(2-3 r) x_{2}-x_{1}+r\left(1-x_{2}\right)\left(r x_{2}-2 r x_{1}+3 x_{1}\right)\right] \\
& \times H_{8}^{(b)}\left(A b_{1}, C_{8} b_{1}, C_{8} b_{2}\right) \\
& +(1+r)\left(1-r^{2}\right)\left[(1+r) x_{1}-r x_{2}\right] H_{8}^{(c)}\left(\sqrt{\left|A^{\prime 2}\right|} b_{2}, D_{8} b_{1}, D_{8} b_{2}\right) \\
& -\left[\left(1-r^{2}\right)\left(\left(1-r^{2}\right)\left(2-x_{2}\right)+(1+3 r)\left(2 x_{2}-x_{1}\right)\right)\right. \\
& \left.\left.+2 r^{2} x_{2}\left(x_{2}-x_{1}\right)\right] H_{8}^{(d)}\left(\sqrt{\left|A^{\prime 2}\right|} b_{1}, E_{8} b_{1}, E_{8} b_{2}\right)\right\}, \\
& M_{8}^{P}=\Gamma^{(0)} \frac{1}{3} \int_{0}^{1} d x_{1} d x_{2} \int_{0}^{1 / \Lambda} b_{1} d b_{1} b_{2} d b_{2} \phi_{B}\left(x_{1}, b_{1}\right) \phi_{K^{*}}\left(x_{2}\right) \\
& \times \alpha_{s}\left(t_{8}\right) C_{8}\left(t_{8}\right) \exp \left[-S_{B}\left(t_{8}\right)-S_{K^{*}}\left(t_{8}\right)\right] \\
& \times\left\{\left(1-r^{2}+x_{1}\right)\left(r x_{2}+x_{1}\right) H_{8}^{(a)}\left(A b_{2}, B_{8} b_{1}, B_{8} b_{2}\right)\right. \\
& +\left[(2-3 r) x_{2}-x_{1}-r\left(1-x_{2}\right)\left(r x_{2}-2 r x_{1}+3 x_{1}\right)\right] \\
& \times H_{8}^{(b)}\left(A b_{1}, C_{8} b_{1}, C_{8} b_{2}\right) \\
& +(1-r)\left(1-r^{2}\right)\left[(1+r) x_{1}-r x_{2}\right] H_{8}^{(c)}\left(\sqrt{\left|A^{\prime 2}\right|} b_{2}, D_{8} b_{1}, D_{8} b_{2}\right) \\
& -\left[\left(1-r^{2}\right)\left(\left(1-r^{2}\right)\left(2+x_{2}\right)-(1-3 r) x_{1}\right)\right.
\end{aligned}
$$




$$
\left.\left.-2 r^{2} x_{2}\left(x_{2}-x_{1}\right)\right] H_{8}^{(d)}\left(\sqrt{\left|A^{\prime 2}\right|} b_{1}, E_{8} b_{1}, E_{8} b_{2}\right)\right\}
$$

with

$$
\begin{aligned}
& A^{\prime 2}=\left(1-r^{2}\right)\left(x_{1}-x_{2}\right) M_{B}^{2}, \quad B_{8}^{2}=\left(1-r^{2}+x_{1}\right) M_{B}^{2}, \\
& C_{8}^{2}=\left(1-x_{2}\right) M_{B}^{2}, \quad D_{8}^{2}=\left(1-r^{2}\right) x_{1} M_{B}^{2}, \quad E_{8}^{2}=\left(1-r^{2}\right) x_{2} M_{B}^{2}, \\
& t_{8}=\max \left(A, \sqrt{\left|A^{\prime 2}\right|}, B_{8}, C_{8}, D_{8}, E_{8}, 1 / b_{1}, 1 / b_{2}\right) .
\end{aligned}
$$

The hard functions are given by

$$
\begin{aligned}
& H_{8}^{(a)}\left(A b_{2}, B_{8} b_{1}, B_{8} b_{2}\right)=H_{7}^{(b)}\left(A b_{2}, B_{8} b_{1}, B_{8} b_{2}\right), \\
& H_{8}^{(b)}\left(A b_{1}, C_{8} b_{1}, C_{8} b_{2}\right)=K_{0}\left(A b_{1}\right) h^{\prime}\left(C_{8} b_{1}, C_{8} b_{2}\right) \\
& H_{8}^{(c)}\left(\sqrt{\left|A^{\prime 2}\right|} b_{2}, D_{8} b_{1}, D_{8} b_{2}\right)= \\
& K_{0}\left(\sqrt{\left|A^{\prime 2}\right|} b_{2}\right) h\left(D_{8} b_{1}, D_{8} b_{2}\right), \quad A^{\prime 2} \geq 0, \\
& i \frac{\pi}{2} H_{0}^{(1)}\left(\sqrt{\left|A^{\prime 2}\right|} b_{2}\right) h\left(D_{8} b_{1}, D_{8} b_{2}\right), \quad A^{\prime 2}<0, \\
& H_{8}^{(d)}\left(\sqrt{\left|A^{\prime 2}\right|} b_{1}, E_{8} b_{1}, E_{8} b_{2}\right)= \\
& K_{0}\left(\sqrt{\left|A^{\prime 2}\right|} b_{1}\right) h^{\prime}\left(E_{8} b_{1}, E_{8} b_{2}\right), \quad A^{\prime 2} \geq 0, \\
& i \frac{\pi}{2} H_{0}^{(1)}\left(\sqrt{\left|A^{\prime 2}\right|} b_{1}\right) h^{\prime}\left(E_{8} b_{1}, E_{8} b_{2}\right), \quad A^{\prime 2}<0 .
\end{aligned}
$$

Compared with the factorization formulas presented in [3], we have included the intrinsic (nonperturbative) $b$ dependence [16] of the $B$ meson wave function $\phi_{B}$. The $b$ dependence appearing in the Sudakov factor is regarded as being perturbative. The intrinsic $b$ dependence, providing additional suppression in the large $b$ region, is more essential for the $B$ meson, in which soft dynamics dominates and Sudakov suppression is weaker. This dependence for the fast recoiling $K^{*}$ meson is less important and neglected. In fact, the intrinsic $b$ dependence of the $K^{*}$ meson wave function is not yet fully understood. For an analysis of a single process such as the decay $B \rightarrow K^{*} \gamma$ [3], the effect of the intrinsic $b$ dependence is not significant, since it can always be compensated by adjusting the $x$ dependence of $\phi_{B}$ under the current accuracy of experimental data. However, this dependence turns out to be 
essential, when one intends to explain data of several $B$ meson decay modes simultaneously. This observation will become clear in Sec. IV.

Another modification is that we have chosen a single hard scale $t$ for all diagrams contributing to an amplitude, which is defined as the maximal scale appearing in these diagrams. In [3] $t$ is chosen as the hard gluon momentum of each diagram, and thus differs among various diagrams contributing to an amplitude. This modification simplifies the factorization formulas for the decay $B \rightarrow K^{*} \gamma$. The difference between these two schemes arises at the level of next-to-next-to-leading logarithms, if $B$ meson decays are indeed dominated by hard gluon exchanges.

\section{THE $B \rightarrow D^{(*)} \pi$ DECAYS}

In this section we quote the factorization formulas for the nonleptonic $B \rightarrow D^{(*)} \pi$ decays, whose effective Hamiltonian is given by

$$
H_{\mathrm{eff}}=\frac{G_{F}}{\sqrt{2}} V_{u d}^{*} V_{c b}\left[C_{1}(\mu) O_{1}(\mu)+C_{2}(\mu) O_{2}(\mu)\right]
$$

with the four-fermion operators

$$
O_{1}=\left(\bar{d}_{i} u_{i}\right)_{V-A}\left(\bar{c}_{j} b_{j}\right)_{V-A}, \quad O_{2}=\left(\bar{d}_{i} u_{j}\right)_{V-A}\left(\bar{c}_{j} b_{i}\right)_{V-A}
$$

Note the interchange of the definitions of $O_{1}$ and $O_{2}$ compared with those in Eq. (2.1). Similarly, we assign the momenta of the $B$ and $D^{(*)}$ mesons as $P_{1}=\left(M_{B} / \sqrt{2}\right)\left(1,1, \mathbf{0}_{T}\right)$ and $P_{2}=\left(M_{B} / \sqrt{2}\right)\left(1, r^{2}, \mathbf{0}_{T}\right)$, respectively, with $r=M_{D^{(*)}} / M_{B}, M_{D^{(*)}}$ being the mass of the $D^{(*)}$ meson. $\epsilon^{*}$ is the polarization vector of the $D^{*}$ meson. The momentum fractions $x_{1}$ and $x_{2}$, and the transverse momenta $\mathbf{k}_{1 T}$ and $\mathbf{k}_{2 T}$ are defined similarly as in Sec. II. The pion momentum $P_{3}=P_{1}-P_{2}$ is the same as the photon momentum in Sec. II. One of its valence quark carries the fractional momentum $x_{3} P_{3}^{-}$, and small transverse momenta $\mathbf{k}_{3 T}$.

In the present analysis we regard the $D^{(*)}$ meson as a heavy meson. Hence, the Sudakov

form factor $\exp \left(-S_{D^{(*)}}\right)$ from the resummation of the double logarithmic corrections to the 
$D^{(*)}$ meson wave function $\phi_{D^{(*)}}$ has the same expression as $\exp \left(-S_{B}\right)$ but with different kinematical variables. The Sudakov form factor $\exp \left(-S_{\pi}\right)$ associated with the pion wave function $\phi_{\pi}$ contains the exponent 17]

$$
S_{\pi}(t)=s\left(x_{3} P_{3}^{-}, b_{3}\right)+s\left(\left(1-x_{3}\right) P_{3}^{-}, b_{3}\right)+2 \int_{1 / b_{3}}^{t} \frac{d \bar{\mu}}{\bar{\mu}} \gamma\left(\alpha_{s}(\bar{\mu})\right) .
$$

The decay rates of $B \rightarrow D^{(*)} \pi$ have the expression

$$
\Gamma_{i}=\frac{1}{128 \pi} G_{F}^{2}\left|V_{c b}\right|^{2}\left|V_{u d}\right|^{2} M_{B}^{3} \frac{\left(1-r^{2}\right)^{3}}{r}\left|\mathcal{M}_{i}\right|^{2},
$$

where $i=1,2,3$, and 4 denote the modes $B^{-} \rightarrow D^{0} \pi^{-}, \bar{B}^{0} \rightarrow D^{+} \pi^{-}, B^{-} \rightarrow D^{* 0} \pi^{-}$, and $\bar{B}^{0} \rightarrow D^{*+} \pi^{-}$, respectively. The decay amplitudes $\mathcal{M}_{i}$ are written as

$$
\begin{aligned}
\mathcal{M}_{1}= & f_{\pi}\left[(1+r) \xi_{+}-(1-r) \xi_{-}\right]+f_{D} \xi_{\mathrm{int}}+\mathcal{M}_{\mathrm{ext}}+\mathcal{M}_{\mathrm{int}}, \\
\mathcal{M}_{2}= & f_{\pi}\left[(1+r) \xi_{+}-(1-r) \xi_{-}\right]+f_{B} \xi_{\mathrm{exc}}+\mathcal{M}_{\mathrm{ext}}+\mathcal{M}_{\mathrm{exc}}, \\
\mathcal{M}_{3}= & \frac{1+r}{2 r} f_{\pi}\left[(1+r) \xi_{A_{1}}-(1-r)\left(r \xi_{A_{2}}+\xi_{A_{3}}\right)\right] \\
& +f_{D^{*}} \xi_{\mathrm{int}}^{*}+\mathcal{M}_{\mathrm{ext}}^{*}+\mathcal{M}_{\mathrm{int}}^{*}, \\
\mathcal{M}_{4}= & \frac{1+r}{2 r} f_{\pi}\left[(1+r) \xi_{A_{1}}-(1-r)\left(r \xi_{A_{2}}+\xi_{A_{3}}\right)\right] \\
& +f_{B} \xi_{\mathrm{exc}}^{*}+\mathcal{M}_{\mathrm{ext}}^{*}+\mathcal{M}_{\mathrm{exc}}^{*},
\end{aligned}
$$

where $f_{B}, f_{D^{(*)}}$, and $f_{\pi}$ are the $B$ meson, $D^{(*)}$ meson, and pion decay constants, respectively. The form factors $\xi_{i}, i=+,-, V, A_{1}, A_{2}$, and $A_{3}$, denote the factorizable external $W$-emission contributions. The form factors $\xi_{\text {int }}^{(*)}$ and $\xi_{\text {exc }}^{(*)}$ denote the factorizable internal $W$-emission and $W$-exchange contributions, respectively. The amplitudes $\mathcal{M}_{\text {ext }}^{(*)}, \mathcal{M}_{\text {int }}^{(*)}$, and $\mathcal{M}_{\text {exc }}^{(*)}$ represent the nonfactorizable external $W$-emission, internal $W$-emission, and $W$ exchange contributions, respectively.

The form factors $\xi_{i}, i=+, A_{1}$ and $A_{3}$, and $\xi_{j}, j=-$ and $A_{2}$, are given by

$$
\begin{aligned}
\xi_{i}= & 16 \pi C_{F} \sqrt{r} M_{B}^{2} \int_{0}^{1} d x_{1} d x_{2} \int_{0}^{\infty} b_{1} d b_{1} b_{2} d b_{2} \phi_{B}\left(x_{1}, b_{1}\right) \phi_{D^{(*)}}\left(x_{2}, b_{2}\right) \\
& \times \alpha_{s}(t) a_{1}(t) \exp \left[-S_{B}(t)-S_{D^{(*)}}(t)\right] \\
& \times\left[\left(1+\zeta_{i} x_{2} r\right) h\left(x_{1}, x_{2}, b_{1}, b_{2}, m\right)+\left(r+\zeta_{i}^{\prime} x_{1}\right) h\left(x_{2}, x_{1}, b_{2}, b_{1}\right)\right],
\end{aligned}
$$




$$
\begin{aligned}
\xi_{j}= & 16 \pi C_{F} \sqrt{r} M_{B}^{2} \int_{0}^{1} d x_{1} d x_{2} \int_{0}^{\infty} b_{1} d b_{1} b_{2} d b_{2} \phi_{B}\left(x_{1}, b_{1}\right) \phi_{D^{(*)}}\left(x_{2}, b_{2}\right) \\
& \times \alpha_{s}(t) a_{1}(t) \exp \left[-S_{B}(t)-S_{D^{(*)}}(t)\right] \\
& \times\left[\zeta_{j} x_{2} r h\left(x_{1}, x_{2}, b_{1}, b_{2}\right)+\zeta_{j}^{\prime} x_{1} h\left(x_{2}, x_{1}, b_{2}, b_{1}\right)\right],
\end{aligned}
$$

with the constants 18

$$
\begin{aligned}
& \zeta_{+}=\zeta_{+}^{\prime}=\frac{1}{2}\left[\eta-\frac{3}{2}+\sqrt{\frac{\eta-1}{\eta+1}}\left(\eta-\frac{1}{2}\right)\right], \\
& \zeta_{-}=-\zeta_{-}^{\prime}=-\frac{1}{2}\left[\eta-\frac{1}{2}+\sqrt{\frac{\eta+1}{\eta-1}}\left(\eta-\frac{3}{2}\right)\right], \\
& \zeta_{A_{1}}=-\frac{2-\eta-\sqrt{\eta^{2}-1}}{2(\eta+1)}, \quad \zeta_{A_{1}}^{\prime}=-\frac{1}{2(\eta+1)}, \\
& \zeta_{A_{2}}=0, \quad \zeta_{A_{2}}^{\prime}=-1-\frac{\eta}{\sqrt{\eta^{2}-1}}, \\
& \zeta_{A_{3}}=-\frac{1}{2}-\frac{\eta-2}{2 \sqrt{\eta^{2}-1}}, \quad \zeta_{A_{3}}^{\prime}=\frac{1}{2 \sqrt{\eta^{2}-1}},
\end{aligned}
$$

where the velocity transfer $\eta$ takes the maximal value $\eta_{\max }=\left(1+r^{2}\right) /(2 r)$ here. The form factors $\xi_{\text {int }}^{(*)}$ and $\xi_{\text {exc }}^{(*)}$ are written as

$$
\begin{aligned}
\xi_{\text {int }}^{(*)}= & 16 \pi C_{F} \sqrt{r} M_{B}^{2} \int_{0}^{1} d x_{1} d x_{3} \int_{0}^{\infty} b_{1} d b_{1} b_{3} d b_{3} \phi_{B}\left(x_{1}, b_{1}\right) \phi_{\pi}\left(x_{3}\right) \\
& \times \alpha_{s}\left(t_{\text {int }}\right) a_{2}\left(t_{\text {int }}\right) \exp \left[-S_{B}\left(t_{\text {int }}\right)-S_{\pi}\left(t_{\text {int }}\right)\right] \\
& \times\left[\left(1+x_{3}\left(1-r^{2}\right)\right) h_{\text {int }}\left(x_{1}, x_{3}, b_{1}, b_{3}, m_{\text {int }}\right)\right. \\
& \left.+\zeta_{\text {int }}^{(*)} x_{1} r^{2} h_{\text {int }}\left(x_{3}, x_{1}, b_{3}, b_{1}, m_{\text {int }}\right)\right] \\
\xi_{\text {exc }}^{(*)}= & 16 \pi C_{F} \sqrt{r} M_{B}^{2} \int_{0}^{1} d x_{2} d x_{3} \int_{0}^{\infty} b_{2} d b_{2} b_{3} d b_{3} \phi_{D^{(*)}}\left(x_{2}, b_{2}\right) \phi_{\pi}\left(x_{3}\right) \\
& \times \alpha_{s}\left(t_{\text {exc }}\right) a_{2}\left(t_{\mathrm{exc}}\right) \exp \left[-S_{D^{(*)}}\left(t_{\mathrm{exc}}\right)-S_{\pi}\left(t_{\mathrm{exc}}\right)\right] \\
& \times\left[\left(x_{3}\left(1-r^{2}\right)-\zeta_{\mathrm{exc}}^{(*)} r^{2}\right) h_{\mathrm{exc}}\left(x_{2}, x_{3}, b_{2}, b_{3}, m_{\mathrm{exc}}\right)\right. \\
& \left.-x_{2} h_{\mathrm{exc}}\left(x_{3}, x_{2}, b_{3}, b_{2}, m_{\mathrm{exc}}\right)\right]
\end{aligned}
$$

with the constants $\zeta_{\text {int }}=\zeta_{\mathrm{exc}}=-\zeta_{\text {int }}^{*}=-\zeta_{\mathrm{exc}}^{*}=1$.

In Eqs. (3.9), (3.10), (3.12), and (3.13) the Wilson coefficients $a_{1}$ and $a_{2}$ are defined by

$$
a_{1}=C_{1}+\frac{C_{2}}{N_{c}}, \quad a_{2}=C_{2}+\frac{C_{1}}{N_{c}},
$$


with $N_{c}=3$ the number of colors. The functions $h$ 's, obtained from the Fourier transformation of the lowest-order $H$, are given by

$$
\begin{aligned}
h\left(x_{1}, x_{2}, b_{1}, b_{2}, m\right)= & K_{0}\left(\sqrt{x_{1} x_{2} m} b_{1}\right) \\
\times & {\left[\theta\left(b_{1}-b_{2}\right) K_{0}\left(\sqrt{x_{2} m} b_{1}\right) I_{0}\left(\sqrt{x_{2} m} b_{2}\right)\right.} \\
& \left.+\theta\left(b_{2}-b_{1}\right) K_{0}\left(\sqrt{x_{2} m} b_{2}\right) I_{0}\left(\sqrt{x_{2} m} b_{1}\right)\right], \\
h_{\text {int }}\left(x_{1}, x_{3}, b_{1}, b_{3}, m_{\text {int }}\right)= & h\left(x_{1}, x_{3}, b_{1}, b_{3}, m_{\text {int }}\right), \\
h_{\mathrm{exc}}\left(x_{2}, x_{3}, b_{2}, b_{3}, m_{\mathrm{exc}}\right)= & \frac{\pi^{2}}{4} H_{0}^{(1)}\left(\sqrt{x_{2} x_{3} m_{\mathrm{exc}}} b_{2}\right) \\
& \times\left[\theta\left(b_{2}-b_{3}\right) H_{0}^{(1)}\left(\sqrt{x_{3} m_{\mathrm{exc}}} b_{2}\right) J_{0}\left(\sqrt{x_{3} m_{\mathrm{exc}}} b_{3}\right)\right. \\
& \left.+\theta\left(b_{3}-b_{2}\right) H_{0}^{(1)}\left(\sqrt{x_{3} m_{\mathrm{exc}}} b_{3}\right) J_{0}\left(\sqrt{x_{3} m_{\mathrm{exc}}} b_{2}\right)\right],
\end{aligned}
$$

with $m=M_{B}^{2}$ and $m_{\text {int }}=m_{\text {exc }}=\left(1-r^{2}\right) M_{B}^{2}$. The hard scales $t$ are chosen as

$$
\begin{aligned}
t & =\max \left(\sqrt{x_{1} m}, \sqrt{x_{2} m}, 1 / b_{1}, 1 / b_{2}\right), \\
t_{\mathrm{int}} & =\max \left(\sqrt{x_{1} m_{\mathrm{int}}}, \sqrt{x_{3} m_{\mathrm{int}}}, 1 / b_{1}, 1 / b_{3}\right), \\
t_{\mathrm{exc}} & =\max \left(\sqrt{x_{2} m_{\mathrm{exc}}}, \sqrt{x_{3} m_{\mathrm{exc}}}, 1 / b_{2}, 1 / b_{3}\right) .
\end{aligned}
$$

For the nonfactorizable amplitudes, the factorization formulas involve the kinematic variables of all the three mesons, and the Sudakov exponent is given by $S=S_{B}+S_{D^{(*)}}+S_{\pi}$. The integration over $b_{3}$ can be performed trivially, leading to $b_{3}=b_{1}$ or $b_{3}=b_{2}$. Their expressions are

$$
\begin{aligned}
\mathcal{M}_{\mathrm{ext}}^{(*)}= & 32 \pi \sqrt{2 N} C_{F} \sqrt{r} M_{B}^{2} \int_{0}^{1}[d x] \int_{0}^{\infty} b_{1} d b_{1} b_{2} d b_{2} \phi_{B}\left(x_{1}, b_{1}\right) \phi_{D^{(*)}}\left(x_{2}, b_{2}\right) \phi_{\pi}\left(x_{3}\right) \\
& \times \alpha_{s}\left(t_{b}\right) \frac{C_{2}\left(t_{b}\right)}{N} \exp \left[-\left.S\left(t_{b}\right)\right|_{\left.b_{2}=b_{1}, b_{3}=b_{2}\right]}\right] \\
\times & \left\{\left[x_{3}\left(1-r^{2}\right)-x_{1}-\zeta_{b}^{(*)} x_{2}\left(r-r^{2}\right)\right] h_{b}^{(1)}\left(x_{i}, b_{i}\right)\right. \\
& \left.\quad-\left[x_{3}\left(1-r^{2}\right)-x_{1}+x_{2}\right] h_{b}^{(2)}\left(x_{i}, b_{i}\right)\right\}, \\
\mathcal{M}_{\text {int }}^{(*)}= & 32 \pi \sqrt{2 N} C_{F} \sqrt{r} M_{B}^{2} \int_{0}^{1}[d x] \int_{0}^{\infty} b_{1} d b_{1} b_{2} d b_{2} \phi_{B}\left(x_{1}, b_{1}\right) \phi_{D^{(*)}}\left(x_{2}, b_{2}\right) \phi_{\pi}\left(x_{3}\right) \\
& \times \alpha_{s}\left(t_{d}\right) \frac{C_{1}\left(t_{d}\right)}{N} \exp \left[-\left.S\left(t_{d}\right)\right|_{b_{3}=b_{1}}\right]
\end{aligned}
$$




$$
\begin{aligned}
\times & \left\{\left[x_{1}-x_{2}-x_{3}\left(1-r^{2}\right)\right] h_{d}^{(1)}\left(x_{i}, b_{i}\right)\right. \\
& \left.-\left[\left(x_{1}+x_{2}\right)\left(1+\zeta_{d}^{(*)} r^{2}\right)-1\right] h_{d}^{(2)}\left(x_{i}, b_{i}\right)\right\} \\
\mathcal{M}_{\mathrm{exc}}^{(*)}= & 32 \pi \sqrt{2 N} C_{F} \sqrt{r} M_{B}^{2} \int_{0}^{1}[d x] \int_{0}^{\infty} b_{1} d b_{1} b_{2} d b_{2} \phi_{B}\left(x_{1}, b_{1}\right) \phi_{D^{(*)}}\left(x_{2}, b_{2}\right) \phi_{\pi}\left(x_{3}\right) \\
\times & \alpha_{s}\left(t_{f}\right) \frac{C_{1}\left(t_{f}\right)}{N} \exp \left[-\left.S\left(t_{f}\right)\right|_{b_{3}=b_{2}}\right] \\
\times & \left\{\left[x_{3}\left(1-r^{2}\right)-\zeta_{f}^{(*)}\left(x_{1}-x_{2}\right) r^{2}\right] h_{f}^{(1)}\left(x_{i}, b_{i}\right)\right. \\
& \left.-\left[\left(x_{1}+x_{2}\right)\left(1+\zeta_{f}^{(*)} r^{2}\right)-\zeta_{f}^{(*)} r^{2}\right] h_{f}^{(2)}\left(x_{i}, b_{i}\right)\right\}
\end{aligned}
$$

with the definition $[d x] \equiv d x_{1} d x_{2} d x_{3}$. The constants are $\zeta_{b, d, f}=-\zeta_{b, d, f}^{*}=1$.

The functions $h^{(j)}, j=1$ and 2, appearing in Eqs. (3.21)-(3.23), are written as

$$
\begin{aligned}
& h_{b}^{(j)}=\left[\theta\left(b_{1}-b_{2}\right) K_{0}\left(B M_{B} b_{1}\right) I_{0}\left(B M_{B} b_{2}\right)\right. \\
& \left.+\theta\left(b_{2}-b_{1}\right) K_{0}\left(B M_{B} b_{2}\right) I_{0}\left(B M_{B} b_{1}\right)\right] \\
& \times\left(\begin{array}{cc}
K_{0}\left(B_{j} M_{B} b_{2}\right) & \text { for } B_{j}^{2} \geq 0 \\
\frac{i \pi}{2} H_{0}^{(1)}\left(\sqrt{\left|B_{j}^{2}\right|} M_{B} b_{2}\right) & \text { for } B_{j}^{2} \leq 0
\end{array}\right), \\
& h_{d}^{(j)}=\left[\theta\left(b_{1}-b_{2}\right) K_{0}\left(D M_{B} b_{1}\right) I_{0}\left(D M_{B} b_{2}\right)\right. \\
& \left.+\theta\left(b_{2}-b_{1}\right) K_{0}\left(D M_{B} b_{2}\right) I_{0}\left(D M_{B} b_{1}\right)\right] \\
& \times\left(\begin{array}{cc}
K_{0}\left(D_{j} M_{B} b_{2}\right) & \text { for } D_{j}^{2} \geq 0 \\
\frac{i \pi}{2} H_{0}^{(1)}\left(\sqrt{\left|D_{j}^{2}\right|} M_{B} b_{2}\right) & \text { for } D_{j}^{2} \leq 0
\end{array}\right), \\
& h_{f}^{(j)}=i \frac{\pi}{2}\left[\theta\left(b_{1}-b_{2}\right) H_{0}^{(1)}\left(F M_{B} b_{1}\right) J_{0}\left(F M_{B} b_{2}\right)\right. \\
& \left.+\theta\left(b_{2}-b_{1}\right) H_{0}^{(1)}\left(F M_{B} b_{2}\right) J_{0}\left(F M_{B} b_{1}\right)\right] \\
& \times\left(\begin{array}{cc}
K_{0}\left(F_{j} M_{B} b_{1}\right) & \text { for } F_{j}^{2} \geq 0 \\
\frac{i \pi}{2} H_{0}^{(1)}\left(\sqrt{\left|F_{j}^{2}\right|} M_{B} b_{1}\right) & \text { for } F_{j}^{2} \leq 0
\end{array}\right),
\end{aligned}
$$

with the variables

$$
\begin{aligned}
& B^{2}=x_{1} x_{2} \\
& B_{1}^{2}=x_{1} x_{2}-x_{2} x_{3}\left(1-r^{2}\right), \\
& B_{2}^{2}=x_{1} x_{2}\left(1+r^{2}\right)-\left(x_{1}-x_{2}\right)\left(1-x_{3}\right)\left(1-r^{2}\right), \\
& D^{2}=x_{1} x_{3}\left(1-r^{2}\right),
\end{aligned}
$$




$$
\begin{aligned}
& D_{1}^{2}=F_{1}^{2}=3 D\left(x_{1}-x_{2}\right) x_{3}\left(1-r^{2}\right), \\
& D_{2}^{2}=\left(x_{1}+x_{2}\right) r^{2}-\left(1-x_{1}-x_{2}\right) x_{3}\left(1-r^{2}\right), \\
& F^{2}=x_{2} x_{3}\left(1-r^{2}\right) \\
& F_{2}^{2}=x_{1}+x_{2}+\left(1-x_{1}-x_{2}\right) x_{3}\left(1-r^{2}\right) .
\end{aligned}
$$

The scales $t^{(j)}$ are chosen as

$$
\begin{aligned}
& t_{b}=\max \left(B M_{B}, \sqrt{\left|B_{1}^{2}\right|} M_{B}, \sqrt{\left|B_{2}^{2}\right|} M_{B}, 1 / b_{1}, 1 / b_{2}\right) \\
& t_{d}=\max \left(D M_{B}, \sqrt{\left|D_{1}^{2}\right|} M_{B}, \sqrt{\left|D_{2}^{2}\right|} M_{B}, 1 / b_{1}, 1 / b_{2}\right) \\
& t_{f}=\max \left(F M_{B}, \sqrt{\left|F_{1}^{2}\right|} M_{B}, \sqrt{\left|F_{2}^{2}\right|} M_{B}, 1 / b_{1}, 1 / b_{2}\right)
\end{aligned}
$$

As before, we have included the intrinsic $b$ dependence of wave functions for the heavy $B$ and $D^{(*)}$ mesons, in which soft dynamics is more important. This dependence for the fast recoiling pion is also neglected, similar to the case of the $K^{*}$ meson, for consistency. The scheme of a single hard scale $t$ for all diagrams contributing to an amplitude has been adopted to simplify the factorization formulas. We have also corrected misprints in Eq. (3.27) appearing in [2].

\section{NUMERICAL ANALYSIS}

In this section we determine the heavy-meson wave functions by fitting the predictions

from the factorization formulas for the $B \rightarrow K^{*} \gamma$ and $B \rightarrow D^{(*)} \pi$ decays to the CLEO data [11,19,20

$$
\begin{aligned}
B\left(B \rightarrow K^{*} \gamma\right) & =(4.2 \pm 0.8 \pm 0.6) \times 10^{-5}, \\
B\left(B^{-} \rightarrow D^{0} \pi^{-}\right) & =(5.0 \pm 0.5 \pm 0.2) \times 10^{-3}, \\
B\left(\bar{B}^{0} \rightarrow D^{+} \pi^{-}\right) & =(3.1 \pm 0.4 \pm 0.2) \times 10^{-3}, \\
B\left(B^{-} \rightarrow D^{* 0} \pi^{-}\right) & =(4.34 \pm 0.33 \pm 0.34 \pm 0.18) \times 10^{-3}, \\
B\left(\bar{B}^{0} \rightarrow D^{*+} \pi^{-}\right) & =(2.81 \pm 0.11 \pm 0.21 \pm 0.05) \times 10^{-3} .
\end{aligned}
$$


We adopt $G_{F}=1.16639 \times 10^{-5} \mathrm{GeV}^{-2}$, the CKM matrix elements $\left|V_{c b}\right|=0.040$ [5, 18] and $\left|V_{u d}\right|=0.974$, the masses $M_{B}=5.28 \mathrm{GeV}, M_{D}=1.87 \mathrm{GeV}$, and $M_{D^{*}}=2.01 \mathrm{GeV}$ [21], and the $\bar{B}^{0}\left(B^{-}\right)$meson lifetime $\tau_{B^{0}}=1.53\left(\tau_{B^{-}}=1.68\right)$ ps [22].

As the transverse extent $b$ approaches zero, $\phi(x, b)$ reduces to the standard parton model $\phi(x)$, i.e., $\phi(x)=\phi(x, b=0)$. The wave functions $\phi_{i}(x), i=B, D, D^{*}, K^{*}$, and $\pi$, satisfy the normalization

$$
\int_{0}^{1} \phi_{i}(x) d x=\frac{f_{i}}{2 \sqrt{6}}
$$

with the corresponding decay constants $f_{i}$. We choose $f_{B}=180 \mathrm{MeV}$ (in the convention $f_{\pi}=132 \mathrm{MeV}$ ), which is reasonable according to the lattice calculations presented in the literature [23]. For the wave function of the $K^{*}$ meson with transverse polarizations, we employ [10]

$$
\phi_{K^{*}}(x)=\frac{\sqrt{6}}{2} f_{K^{*}} x(1-x)\left[1+0.6(1-2 x)+0.06\left(5(1-2 x)^{2}-1\right)\right],
$$

with $f_{K^{*}}=185 \mathrm{MeV}$. As to the pion wave function, we employ the asymptotic form,

$$
\phi_{\pi}(x)=\frac{\sqrt{6}}{2} f_{\pi} x(1-x)
$$

which has been found to give predictions consistent with the data of the $\pi \gamma$ form factor [24].

For the $B$ meson wave functions, we use the model [25]

$$
\phi_{B}(x, b)=N_{B} \sqrt{x(1-x)} \exp \left[-\frac{1}{2}\left(\frac{x M_{B}}{\omega_{B}}\right)^{2}-\frac{\omega_{B}^{2} b^{2}}{2}\right],
$$

where $N_{B}$ is the normalization constant and $\omega_{B}$ the shape parameter. Another model with a flat profile [26]

$$
\phi_{B}^{\prime}(x, b)=\frac{N_{B}^{\prime} b x^{2}(1-x)^{2}}{\sqrt{M_{B}^{2} x+C_{B} x(1-x)}} K_{1}\left(\sqrt{M_{B}^{2} x+C_{B} x(1-x)} b\right),
$$

gives predictions for the branching ratio of the $B \rightarrow K^{*} \gamma$ decay, which are too small compared with the data. Therefore, we shall not consider it in the numerical analysis below. For the $D^{(*)}$ meson wave functions, we adopt the same model as of the $B$ meson in Eq. (4.5), but with different normalization constants $N_{D^{(*)}}$ and shape parameters $\omega_{D^{(*)}}$, 


$$
\phi_{D^{(*)}}(x, b)=N_{D^{(*)}} \sqrt{x(1-x)} \exp \left[-\frac{1}{2}\left(\frac{x M_{D^{(*)}}}{\omega_{D^{(*)}}}\right)^{2}-\frac{\omega_{D^{(*)}}^{2} b^{2}}{2}\right] .
$$

We shall determine 5 parameters $N_{D}\left(f_{D}\right), N_{D^{*}}\left(f_{D^{*}}\right), \omega_{B}, \omega_{D}$, and $\omega_{D^{*}}$ from the data of the 5 processes in Eq. (4.1), under the constraints $f_{D^{(*)}}>f_{B}$, which is reasonable from the viewpoint of heavy quark symmetry. The fitting procedures are as follows. Start with the $K^{*}$ meson wave function in Eq. (4.3), and determine the $B$ meson wave function (i.e., the shape parameter $\left.\omega_{B}\right)$ from the data of the decay $B \rightarrow K^{*} \gamma$. The obtained $B$ meson wave function is then employed to evaluate the branching ratios of the charged and neutral $B$ decays $B \rightarrow D^{(*)} \pi$, from which both parameters, $N_{D^{(*)}}$ and $\omega_{D^{(*)}}$, of the $D^{(*)}$ meson wave function can be extracted. We have tested the heavy meson wave functions without the intrinsic $b$ dependence. It turns out that the $D^{(*)}$ meson wave function $\phi_{D^{(*)}}(x)$, along with the $B$ meson wave function $\phi_{B}(x)$ determined from the $B \rightarrow K^{*} \gamma$ data, always lead to predictions for the $B \rightarrow D^{(*)} \pi$ decays which exceed the data by a factor 3 , no matter how $\phi_{D^{(*)}}(x)$ is adjusted under the constraint $f_{D^{(*)}}>f_{B}$. These predictions are lowered only by introducing the intrinsic $b$ dependence into heavy meson wave functions, implying that the intrinsic $b$ dependence is indeed essential.

It is easy to understand that the parameters $\omega_{D^{(*)}}$ control the difference between the charged and neutral $B$ meson decay rates. This fact is obvious from Eqs. (3.5)-(3.8): by varying $\omega_{D^{(*)}}$, the external $W$-emission contributions, i.e., the $B \rightarrow D$ form factors change, while the internal $W$-emission contributions, i.e., the $B \rightarrow \pi$ form factors remain the same. The $W$-exchange and nonfactorizable diagrams give smaller contributions than the $W$-emission diagrams to the decays considered. Therefore, the charged $B$ decay rates, as a combination of the external and internal $W$-emission contributions, and the neutral $B$-meson decay rates with the external $W$ emissions as the main contribution, vary differently with the change of $\omega_{D^{(*)}}$.

After a tedious numerical analysis, we determine the meson wave functions,

$$
\phi_{B}(x, b)=1.44605 \sqrt{x(1-x)} \exp \left[-\frac{1}{2}\left(\frac{x M_{B}}{0.462 \mathrm{GeV}}\right)^{2}-\frac{1}{2}(0.462 \mathrm{GeV})^{2} b^{2}\right] \text {, }
$$




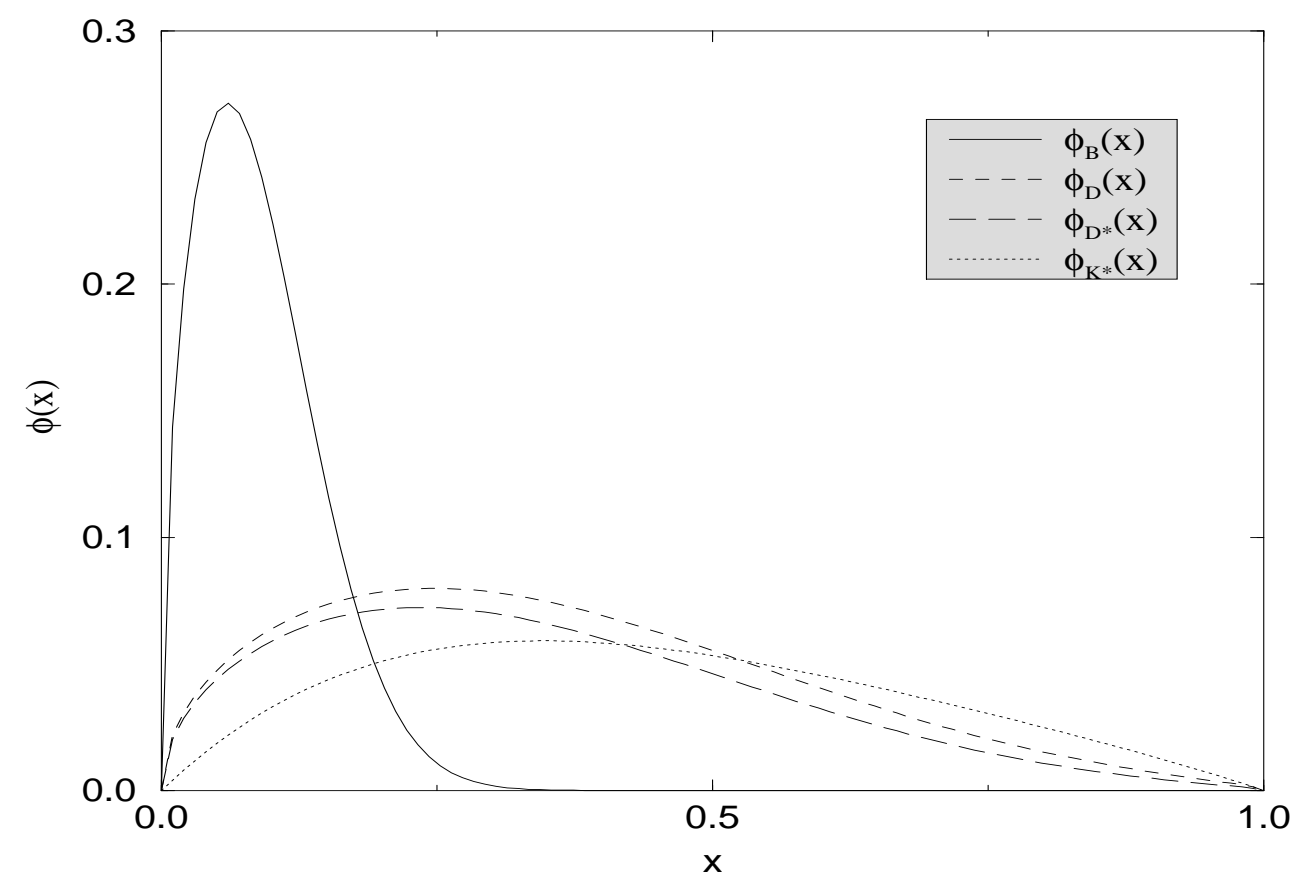

FIG. 1. The $K^{*}, B$ and $D^{(*)}$ meson wave functions.

$$
\begin{gathered}
\phi_{D}(x, b)=0.218677 \sqrt{x(1-x)} \exp \left[-\frac{1}{2}\left(\frac{x M_{D}}{0.8 \mathrm{GeV}}\right)^{2}-\frac{1}{2}(0.8 \mathrm{GeV})^{2} b^{2}\right] \\
\phi_{D^{*}}(x, b)=0.202997 \sqrt{x(1-x)} \exp \left[-\frac{1}{2}\left(\frac{x M_{D^{*}}}{0.8 \mathrm{GeV}}\right)^{2}-\frac{1}{2}(0.8 \mathrm{GeV})^{2} b^{2}\right]
\end{gathered}
$$

which correspond to the decay constants $f_{B}=180 \mathrm{MeV}, f_{D}=220 \mathrm{MeV}$, and $f_{D^{*}}=190$ $\mathrm{MeV}$, respectively. With these heavy meson wave functions, we obtain

$$
\begin{aligned}
B\left(B \rightarrow K^{*} \gamma\right) & =4.19 \times 10^{-5}, \\
B\left(B^{-} \rightarrow D^{0} \pi^{-}\right) & =4.74 \times 10^{-3}, \\
B\left(\bar{B}^{0} \rightarrow D^{+} \pi^{-}\right) & =3.50 \times 10^{-3}, \\
B\left(B^{-} \rightarrow D^{* 0} \pi^{-}\right) & =3.93 \times 10^{-3}, \\
B\left(\bar{B}^{0} \rightarrow D^{*+} \pi^{-}\right) & =2.94 \times 10^{-3},
\end{aligned}
$$

which are consistent with the data in Eq. (4.1). Note that the value $\omega_{B}=0.462 \mathrm{GeV}$ is in good agreement with $\omega_{B}=0.4 \mathrm{GeV}$ obtained in the oscillating quark model [25]. It indicates that meson wave functions in PQCD factorization theorems have absorbed nonperturbative dynamics in $B$ meson decays appropriately, and that remained dynamics, collected by the 
hard quark-level amplitudes, is perturbative. The $x$ dependence of the extracted heavy meson wave functions and of the $K^{*}$ meson wave function is displayed in Fig. 1. As expected, the maximum of a heavy meson wave function moves to the large $x$ region as the meson mass decreases.

\section{CONCLUSION}

In this paper we have extracted heavy meson wave functions, as shown in Eq. (4.8), from the experimental data of the $B \rightarrow K^{*} \gamma$ and $B \rightarrow D^{(*)} \pi$ decays. The extracted meson decay constants $f_{D}$ and $f_{D^{*}}$, compared to $f_{B}$, and the behavior of the wave functions exhibited in Fig. 1 are all reasonable. We have also confirmed that the intrinsic $b$ dependence of heavy meson wave functions, which makes possible a simultaneous fit to the data of several decay modes, is essential. Although the uncertainties of the data are still large, such that wide ranges of the relevant parameters are allowed, we shall employ the obtained wave functions to make predictions for other nonleptonic $B$ meson decay modes, especially charmless de-

cays. As the precision of data improves, the heavy meson wave functions determined in our formalism will be more reliable. On the other hand, we can also proceed with a global determination of the heavy meson wave functions by incorporating more decay modes into the fitting procedures. These subjects will be discussed elsewhere.

\section{ACKNOWLEDGMENTS}

We thank P. Ball for useful comments and discussions. This work was supported by the National Science Council of R.O.C. under the Grant No. NSC-88-2112-M-006-013 and by the Ministry of Science and Technology of the Republic of Croatia under Contract No. 00980102. 


\section{REFERENCES}

[1] C.H. Chang and H-n. Li, Phys. Rev. D 55, 5577 (1997).

[2] T.W. Yeh and H-n. Li, Phys. Rev. D 56, 1615 (1997).

[3] H-n. Li and G.L. Lin, preprint NCKU-HEP-98-14 and hep-ph/9812508, to appear in Phys. Rev. D.

[4] H-n. Li and H.L. Yu, Phys. Rev. Lett. 74, 4388 (1995); Phys. Lett. B 353, 301 (1995); Phys. Rev. D 53, 2480 (1996).

[5] H-n. Li, Phys. Rev. D 52, 3958 (1995).

[6] C. Corianò and H-n. Li, Nucl. Phys. B434, 535 (1995).

[7] P. Ball, J. High Energy Phys. 9809, 005 (1998).

[8] V.L. Chernyak and A.R. Zhitnitsky, Phys. Rep. 112, 173 (1984).

[9] M. Benayoun and V.L. Chernyak, Nucl. Phys. B329, 285 (1990).

[10] P. Ball, V.M. Bauer, Y. Koike, K. Tanaka, Nucl. Phys. B529, 323 (1998).

[11] CLEO Collaboration, R. Ammar et al., "Radiative Penguin Decays of the B Meson," CONF 96-5, ICHEP-96 PA05-9 (1996).

[12] For a review on earlier literatures, see G. Buchalla, A. J. Buras and M. E. Lautenbacher, Review of Modern Physics, 68, 1125 (1996).

[13] J. Milana, Phys. Rev. D 53, 1403 (1996).

[14] J.C. Collins and D.E. Soper, Nucl. Phys. B193, 381 (1981).

[15] J. Botts and G. Sterman, Nucl. Phys. B325, 62 (1989).

[16] M. Dahm, R. Jakob, and P. Kroll, Z. Phys. C 68, 595, (1995).

[17] H-n. Li and G. Sterman Nucl. Phys. B381, 129 (1992). 
[18] C.Y. Wu, T.W. Yeh, and H-n. Li, Phys. Rev. D 53, 4982 (1996).

[19] T.E. Browder, K. Honscheid, and D. Pedrini, Ann. Rev. Nucl. Part. Sci. 46, 395 (1996).

[20] CLEO Collaboration, G. Brandenburg et. al., Phys. Rev. Lett. 80, 2762 (1998).

[21] Particle Data Group, L. Montanet et al, Phys. Rev. D 50, 1173 (1994).

[22] CLEO Collaboration, B. Barish et al., Phys. Rev. D 51, 1014 (1995).

[23] A. Ali Khan, Nucl. Phys. Proc. Suppl. 63, 71 (1998).

[24] P. Kroll and M. Raulfs, Phys. Lett. B 387, 848 (1996); S.J. Brodsky, C.-R. Ji, A. Pang, and D.G. Robertson, Phys. Rev. D 57, 245 (1998); I.V. Musatov and A.V. Radyushkin, Phys. Rev. D 56, 2713 (1997).

[25] M. Bauer and M. Wirbel, Z. Phys. C 42, 671 (1989).

[26] F. Schlumpf, Ph.D. thesis (Zürich U.) and hep-ph/9211255; R. Akhoury, G. Sterman and Y.-P Yao, Phys. Rev. D 50, 358 (1994). 